\title{
Reversals of magnetization and exchange-bias in perovskite chromite $\mathrm{TmCrO}_{3}$
}

\author{
Lei Wang, ${ }^{1,2,3}$ G. H. Rao, ${ }^{2,3 *}$ X. Zhang, ${ }^{2,3}$ L. L. Zhang, ${ }^{4}$ S. W. Wang, ${ }^{2,3}$ Q. R. Yao ${ }^{2,3}$ \\ ${ }^{1}$ School of Materials Science and Engineering, Central South University, Changsha 410083
}

, China

${ }^{2}$ School of Materials Science and Engineering, Guilin University of Electronic Technology,

Guilin 541004, China

${ }^{3}$ Guangxi Key Laboratory of Information Materials, Guilin University of Electronic Technology, Guilin 541004, China

${ }^{4}$ Guangxi Key Laboratory of Superhard Materials, Guilin 541004, China

\begin{abstract}
Reversals of magnetization and exchange bias field were observed in polycrystalline perovskite $\mathrm{TmCrO}_{3}$. Interestingly, the sign reversal of exchange bias can be driven by both temperature and cooling field. The temperature dependence of exchange bias reversal takes place around $31 \mathrm{~K}$ and the sign reversal of exchange bias driven by cooling field occurs when the system exhibits positive exchange bias. The reversals can be elucidated based on the antiferromagnetic coupling between the canted $\mathrm{Cr}^{3+}$ moments and $\mathrm{Tm}^{3+}$ moments. This antiferromagnetic coupling will hinder or benefit the reversal of $\mathrm{Cr}^{3+}$ moments or $\mathrm{Tm}^{3+}$ moments when the system were cooled under different magnetic field.
\end{abstract}

Keywords: Magnetization reversal; Exchange bias reversal; Magnetism

PACS numbers: 75.60.Jk; 75.47.Lx; 75.50.Ee

*corresponding author: rgh@guet.edu.cn 


\section{Introduction}

Exchange bias (EB) effect has attracted a great amount of attention owing to its potential applications in spin-valve devices and in magnetic random access memories (MRAM). The EB initially occurs in slightly oxidized cobalt particles by Meiklejohn and Bean in 1956 [1]. It is defined as a shift of the magnetic hysteresis loop along the field axis for a system cooled down in static magnetic field from above the Néel temperature $\left(T_{N}\right)$ of the antiferromagnet. Usually, a negative exchange bias (NEB) is observed, i.e. the hysteresis loop is shifted to the negative direction of $\mathrm{H}$ axis. EB with hysteresis loop shifted to positive direction of $\mathrm{H}$ axis, on the other hand, is termed as positive exchange bias (PEB). PEB was first observed in $\mathrm{FeF}_{2}-\mathrm{Fe}$ bilayers by Nogués et al. [2], and Koon presented calculations of exchange bias in thin films for explaining it [3]. As the sign reversal of the exchange bias field $\left(H_{E}\right)$ between the NEB and PEB has great deal of applications, it arouses intense interest of the researchers. The sign reversal of EB was reported in many heterostructure systems [2-5] and even in structurally single-phase alloys and compounds [6-9].

It is commonly announced that the magnetic coupling is ferromagnetic at the FM-AFM interface when the system exhibits NEB, and a necessary condition for PEB is an antiferromagnetic coupling at the FM-AFM interface [4]. In this paper, we report the EB reversal driven by temperature and cooling field in $\mathrm{TmCrO}_{3}$. Both of the NEB and PEB were illustrated based on antiferromagnetic coupling between FM component of the canted $\mathrm{Cr}^{3+}$ moments $\left(\mathrm{M}_{\mathrm{Cr}}\right)$ and $\mathrm{Tm}^{3+}$ moments $\left(\mathrm{M}_{\mathrm{Tm}}\right)$. The antiferromagnetic coupling between $\mathrm{Tm}^{3+}$ and $\mathrm{Cr}^{3+}$ moments will hinder or benefit the reversal of $\mathrm{M}_{\mathrm{Cr}}$ or $\mathrm{M}_{\mathrm{Tm}}$ when the system 
were cooled under different field from above $T_{N}$ to a specific temperature.

Magnetization reversal had been observed in some of perovskite chromite $\mathrm{RCrO}_{3}$ [10,11]. The widely accepted mechanism model of magnetization reversal is due to the antiferromagnetic coupling between the $\mathrm{R}^{3+}$ moments and $\mathrm{Cr}^{3+}$ moments. The $\mathrm{R}$ sublattice moments is induced by the antiparallel internal field of the canted $\mathrm{Cr}^{3+}$ spins. Interestingly, magnetization reversal and EB reversal were observed together frequently [8,9]. In our previous work [12], we have highlighted the intrinsic association of the two reversals, and the explanation model in $\mathrm{YbCrO}_{3}$ can also be applicable to $\mathrm{TmCrO}_{3}$.

\section{Experimental}

Polycrystalline sample of perovskite chromite $\mathrm{TmCrO}_{3}$ were prepared with the conventional solid state reaction method. High purity $\mathrm{Tm}_{2} \mathrm{O}_{3}(99.99 \%)$, and $\mathrm{Cr}_{2} \mathrm{O}_{3}(99.9 \%)$ powders were mixed according to atomic ratio of $\mathrm{Tm}$ : $\mathrm{Cr}=1: 1$. And then, the mixed powder was pressed into pellets and sintered at $1400{ }^{\circ} \mathrm{C}$ for 48 hours in the air. The sintering process might be repeated until the sample was identified as single phase by X-ray powder diffraction (XRD). Room temperature XRD measurement was carried out on

a PANalytical X-ray diffractometer with $\mathrm{Cu} K \alpha 1$ radiation $(\lambda=1.54056 \AA$ ). Magnetic measurements were performed on a physical properties measurement system (PPMS 9 T, Quantum Design).

\section{Results and discussion}

Figure 1 shows the XRD pattern and the Rietveld refinement results of the crystal structure of polycrystalline $\mathrm{TmCrO}_{3}$ with the goodness-of-fitting $\chi^{2}=2.64$. All peaks can be indexed by an orthorhombic structure with Pbnm space group with the lattice parameters of 
$a=5.19672(6) \AA, b=5.49182(6) \AA$, and $c=7.48290(4) \AA$, respectively. The details of structural parameters of $\mathrm{TmCrO}_{3}$ were presented in Table 1, indicating a reasonable structural model.

Figure 2 shows the magnetization-temperature curve $(M \sim T)$ of polycrystalline perovskite chromite $\mathrm{TmCrO}_{3}$. The sample was pre-cooled from $300 \mathrm{~K}$ to $5 \mathrm{~K}$ in zero-field (ZFC) or in an applied field of $100 \mathrm{Oe}(\mathrm{FC})$, and then measured from $5 \mathrm{~K}$ to $200 \mathrm{~K}$ under 100 Oe. The inset (a) of Figure 2 shows the reciprocal of the dc magnetic susceptibility vs temperature $\left(\chi^{-1} \sim \mathrm{T}\right)$. The reciprocal of the dc magnetic susceptibility varies linearly with temperature from $135 \mathrm{~K}\left(>T_{N} \approx 125 \mathrm{~K}\right)$ to $200 \mathrm{~K}$, obeying the Curie-Weiss law. The effective magnetic moment calculated based on Curie constant is $8.95 \mu_{\mathrm{B}} / \mathrm{f} . \mathrm{u}$. and the Curie-Weiss temperature is about $-45 \mathrm{~K}$. The calculated effective magnetic moment is close to the theoretical one $\left(8.50 \mu_{\mathrm{B}} / \mathrm{f}\right.$.u. $)$. The inset (b) of Figure 2 shows the $M \sim T$ curve measured under an applied field of $10 \mathrm{kOe}$, which indicates that the magnetization reversal disappears when the cooling field increases to $10 \mathrm{kOe}$. Previous studies on $\mathrm{TmCrO}_{3}$ indicate that the $\mathrm{Cr}^{3+}$ moments ordered in a canted antiferromagnetic structure with a weak ferromagnetic moment below $T_{N}=125 \mathrm{~K}$ and the magnetic structure of the compound is $\Gamma_{2}$ $\left(\begin{array}{lllll}F_{x} & C_{y} & G_{z} ; & F_{x}^{R} C_{y}^{R}\end{array}\right)[13,14]$. During FC measurement, The sample exhibits negative magnetization at low temperature, and the magnetization gradually changes to positive as temperature increases. The compensation temperature $\left(T_{\text {comp }}\right)$ is around $28 \mathrm{~K}$. The negative magnetization of $\mathrm{TmCrO}_{3}$ should be due to the AFM coupling between $\mathrm{Tm}^{3+}$ and $\mathrm{Cr}^{3+}$ sublattices, as illustrated by Shukla [10] and Cao [11], Cr sublattice moments order at $T_{N}$ and impose a negative internal field over the $\mathrm{R}^{3+}$ moments. Therefore, Tm sublattice 
experiences a composite field, which is the sum of the internal field due to Cr sublattice and the external field, and the total magnetization is a superposition of the magnetizations from Cr sublattice and Tm sublattice. Figure 3(a) manifests the coupling scheme of the $\mathrm{Cr}^{3+}$ moments $\left(\mathrm{M}_{\mathrm{Cr}}\right)$ and $\mathrm{Tm}^{3+}$ moments $\left(\mathrm{M}_{\mathrm{Tm}}\right)$ at different temperature regions, where $\mathrm{H}$ is the applied magnetic field and $\mathrm{H}_{\mathrm{I}}$ is the internal field due to the canted AFM ordered $\mathrm{Cr}^{3+}$ spins, respectively. When the temperature is higher than $T_{\text {comp }}\left(T>T_{\text {comp }}\right)$, the $\mathrm{M}_{\mathrm{Cr}}$ dominates over $\mathrm{M}_{\mathrm{Tm}}\left(\mathrm{M}_{\mathrm{Cr}}>\mathrm{M}_{\mathrm{Tm}}\right)$ and this leads to a positive magnetization. Below the $T_{\max }, \mathrm{M}_{\mathrm{Cr}}$ varies slightly with temperature, while $\mathrm{M}_{\mathrm{Tm}}$ keeps increase with decreasing temperature, leading to the decrease of total magnetization. $\mathrm{M}_{\mathrm{Tm}}$ is equal but antiparallel to the $\mathrm{M}_{\mathrm{Cr}}$ at $T_{\text {comp }}=28$ $\mathrm{K}$. When the temperature is lower than $T_{\text {comp }}\left(T<T_{\text {comp }}\right)$, the $\mathrm{M}_{\mathrm{Tm}}$ exceeds the $\mathrm{M}_{\mathrm{Cr}}\left(\mathrm{M}_{\mathrm{Tm}}>\right.$ $\mathrm{M}_{\mathrm{Cr}}$ ), giving rise to a negative magnetization. In contrast, during ZFC measurement (data collected in warming process), $\mathbf{M}_{\mathrm{Tm}}$ dominates over $\mathrm{M}_{\mathrm{Cr}}$ at low temperature, and $\mathbf{M}_{\mathrm{Tm}}$ aligns to the measuring field. Therefore, the antiferromagnetically coupling $\mathrm{M}_{\mathrm{Tm}}$ and $\mathrm{M}_{\mathrm{Cr}}$ were just in the opposite directions compared to those in FC mode [11]. The disappeared magnetization reversal in Figure 2 (b) indicates that when the cooling field increases to 10 kOe (exceeds the negative internal field), the $\mathrm{Tm}^{3+}$ moment will flip to the direction of the external field[11,12]. As reported earlier, the minimum value of magnetization (at $\sim 10 \mathrm{~K}$ ) should be brought about by the spin reorientation of $\mathrm{Tm}^{3+}[14]$.

The magnetization reversal driven by temperature triggers an interest in magnetic field response of the magnetization of $\mathrm{TmCrO}_{3}$ in different temperature regions. Interestingly, the EB effect was observed in this system. In order to investigate details about exchange bias field $\left(H_{E}\right)$ in the compound, the $M \sim H$ loops at different temperature were measured 
(the range of measuring field is $\pm 40 \mathrm{kOe}$ ) and the sample was cooled down from $200 \mathrm{~K}$ to the specific temperature under $10 \mathrm{kOe}$ before each measurement. Figure 4(a) shows the temperature dependence of $H_{E}$. The $H_{E}$ is defined as $H_{E}=\left(H_{1}+H_{2}\right) / 2$, where $H_{1}$ and $H_{2}$ are the right and left coercivity fields in the $M \sim H$ curve [5]. NEB and PEB are observed at high temperature and at low temperature, respectively. The EB reversal occurs around $31 \mathrm{~K}$ $\left(T_{0}\right)$ and there are two blocking temperatures $\left(T_{B} \approx 10 \mathrm{~K}\right.$ and $\left.T_{B}{ }^{\prime} \approx 88 \mathrm{~K}\right)$ in $H_{E}(\mathrm{~T})$. Figure 4(b) shows the temperature dependence of coercivity field $\left(H_{C}\right)$. The $H_{C}$ is defined as $H_{C}=$ $\left(H_{1}-H_{2}\right) / 2$. There are two peaks and one valley in $H_{C}(\mathrm{~T})$ which are perfectly coincident with the two blocking temperature $\left(T_{B}\right.$ and $\left.T_{B}{ }^{\prime}\right)$ and the $T_{0}$ as shown in $H_{E}(\mathrm{~T})$, respectively.

The temperature-driven EB reversal could be attributed to the coupling between the $\mathrm{M}_{\mathrm{Cr}}$ and the antiparallel $\mathrm{M}_{\mathrm{Tm}}$. As we discussed for the magnetization reversal, the dominant $\mathrm{M}_{\mathrm{Cr}}$ or $\mathrm{M}_{\mathrm{Tm}}$ determines the major properties of the system. The AFM coupling between the $\mathrm{M}_{\mathrm{Cr}}$ and $\mathrm{M}_{\mathrm{Tm}}$ will hinder the reversal of the greater one of $\mathrm{M}_{\mathrm{Cr}}$ or $\mathrm{M}_{\mathrm{Tm}}$. When the temperature $T>31 \mathrm{~K}, \mathrm{M}_{\mathrm{Cr}}$ dominates over $\mathrm{M}_{\mathrm{Tm}}$ and $\mathrm{Tm}^{3+} / \mathrm{Cr}^{3+}$ coupled antiferromagnetically. It can be visualized that, during demagnetization process, the AFM coupling between the $\mathrm{M}_{\mathrm{Cr}}$ and $\mathrm{M}_{\mathrm{Tm}}$ will hinder the reversal of $\mathrm{M}_{\mathrm{Cr}}$ and an additional antiparallel applied field $\left(H_{E}\right)$ is required to bring the magnetization towards zero, so the hysteresis loops shift to negative direction of $\mathrm{H}$ axis. When the temperature $T<31 \mathrm{~K}$, $\mathrm{M}_{\mathrm{Tm}}$ dominates over $\mathrm{M}_{\mathrm{Cr}}$, the antiparallel $\mathrm{M}_{\mathrm{Tm}}$ will flip to the direction of the applied field and become parallel to $\mathrm{M}_{\mathrm{Cr}}$ with the increase of measuring field. During demagnetization process, the AFM character of coupling between Tm sublattice and Cr sublattice benefits the reversal of $\mathrm{M}_{\mathrm{Tm}}$, leading to a smaller applied field along the negative direction to 
achieve zero magnetization. Therefore, the hysteresis loops shift to positive direction of $\mathrm{H}$ axis.

The collapse in the width of the $M \sim H$ loop at $T_{\text {comp }}$ of $H_{E}(\mathrm{~T})$ had been observed in single crystal of $\mathrm{Nd}_{0.75} \mathrm{Ho}_{0.25} \mathrm{Al}_{2}$ [8], polycrystalline $\mathrm{LaCr}_{0.85} \mathrm{Mn}_{0.15} \mathrm{O}_{3}$ [9], etc. Webb et al. [15] had argued that in ferrimagnets the drop in coercive field should commence when temperature is close to $T_{\text {comp. }}$. Therefore, the valley in $H_{C}(\mathrm{~T})$ of $\mathrm{TmCrO}_{3}$ should be attributed to the competition between the $\mathrm{M}_{\mathrm{Cr}}$ and antiparallel polarized $\mathrm{M}_{\mathrm{Tm}}$. As we discussed above, the $\mathrm{M}_{\mathrm{Cr}}$ varies slightly when the temperature is below $T_{\max }$, while $\mathrm{M}_{\mathrm{Tm}}$ keeps increase with decreasing temperature till the spin reorientation temperature $\left(T_{S R}\right)$. Thus, one may expect that the EB effect can set in at $T_{B}(\sim 88 \mathrm{~K})$, when the pinning by the AFM coupled interfacial $\mathrm{Tm}^{3+}$ moment becomes strong enough. The EB effect blocked at $T_{B}{ }^{\prime}(\sim 10 \mathrm{~K})$ could be attributed to the spin reorientation of the $\mathrm{Tm}^{3+}$ moments. The spin configuration gradually changes from $F^{R}{ }_{x} C^{R}{ }_{y}$ to $F^{R}$ [13] while the temperature decreases from $\sim 21 \mathrm{~K}$ to $\sim 10 \mathrm{~K}$. This change rotates the direction of the $\mathrm{Tm}^{3+}$ moments by 90 degrees which makes the $\mathrm{M}_{\mathrm{Cr}}$ and $\mathrm{M}_{\mathrm{Tm}}$ away from collinear. Corresponding to the inset of Fig. 4(b), the $\mathrm{dM} / \mathrm{dT}$ changed sharply around $21 \mathrm{~K}$. This feature could be identically due to the spin reorientation of $\mathrm{Tm}^{3+}$ spins. Since the coupling between the $\mathrm{M}_{\mathrm{Cr}}$ and $\mathrm{M}_{\mathrm{Tm}}$ weakened at the blocking temperature, each of them is allowed to order independently. In other words, one component would not strongly affected by the molecular field of the other, leading to the two peaks in $H_{C}(\mathrm{~T})$.

For a further understanding of the $\mathrm{EB}$ effect in perovskite chromite $\mathrm{TmCrO}_{3}$, we studied the cooling field dependence of the EB at different temperatures. The $M \sim H$ 
hysteresis loops were measured between $\pm 40 \mathrm{KOe}$ after the sample of $\mathrm{TmCrO}_{3}$ was cooled under different field from $200 \mathrm{~K}$ to the specific temperatures. As shown in Figure 5, there is no significant change of $H_{E}$ at $10 \mathrm{~K}, 40 \mathrm{~K}$ and $50 \mathrm{~K}$. The EB reversal driven by cooling field takes place when the system is PEB $(20 \mathrm{~K}, 22 \mathrm{~K}, 25 \mathrm{~K}$ and $30 \mathrm{~K}$,) but there is only irregularly fluctuation when the system is NEB (40 K and $50 \mathrm{~K}$ ). It is similar to the observations in $\mathrm{FeF}_{2}-\mathrm{Fe}$ and $\mathrm{MnF}_{2}-\mathrm{Fe}$ bilayers [4] and polycrystalline $\mathrm{La}_{1-\mathrm{x}} \mathrm{Pr}_{\mathrm{x}} \mathrm{CrO}_{3}$ [6]. Figure 3(b) manifests the coupling scheme of the $\mathrm{Cr}^{3+}$ moments and the $\mathrm{Tm}^{3+}$ moments after cooling under low and high field. As we discussed above, when the system was cooled under low field, the AFM character of the coupling between $\mathrm{Tm}^{3+}$ and $\mathrm{Cr}^{3+}$ benefits the reversal of $\mathrm{M}_{\mathrm{Tm}}$ during the demagnetization process, leading to a PEB. In contrast, when the compound was cooled under high field, the $\mathrm{M}_{\mathrm{Cr}}$ and $\mathrm{M}_{\mathrm{Tm}}$ were forced to align parallel with each other. Therefore, this parallel coupling status will hinder the reversal of $\mathrm{M}_{\mathrm{Tm}}$ during the demagnetization process, leading to a NEB. However, this parallel statue is unstable and is compelled by the high cooling field due to the fact that the AFM exchange coupling energy became lower than Zeeman energy[2, 11].

\section{Conclusions}

In summary, the sign reversal of EB driven by both temperature and cooling field in the polycrystalline $\mathrm{TmCrO}_{3}$ were observed. The elucidation of the observed negative EB and positive EB is given based on the AFM coupling between the $\mathrm{M}_{\mathrm{Cr}}$ and $\mathrm{M}_{\mathrm{Tm}}$ which makes $\mathrm{TmCrO}_{3}$ distinguished from other exchange bias systems. The sign reversal of the $H_{E}$ driven by temperature is the main principle to design a thermally assisted magnetic random access memory (TAMRAM) [16] and the switching between NEB and PEB driven 
by cooling field could be used to design spin-valve devices, magnetic tunnel junctions, etc. [17]. Since both temperature and cooling field can drive the EB reversal, our work is expected to generate a considerable interest among researchers working on films or multilayers to realize a sign reversal of EB and to have more choices for implementing this physical effect in future spintronic devices.

\section{Acknowledgments}

This work was supported by the National Science Foundation of China (Grant No. 11464007), National Science Foundation of Guangxi (Grant No. 2012GXNSFGA060002 and 2014GXNSFBA118241), Guangxi Key Laboratory of Information Material Foundation (Grant No. 131021-Z), Guangxi Department of Education Foundation (Grant No. YB2014120 and KY2015YB104).

\section{References}

[1] W. P. Meiklejohn, C. P. Bean, New Magnetic Anisotropy, Phys. Rev. 102 (1956) $1413-1414$.

[2] J. Nogués, D. Lederman, T. J. Moran, I. K. Schuller, Positive Exchange Bias in $\mathrm{FeF}_{2}$-Fe Bilayers, Phys. Rev. Lett. 76 (1996) 4624-4627

[3] N. C. Koon, Calculations of Exchange Bias in Thin Films with Ferromagneticy/Antiferromagnetic Interfaces, Phys. Rev. Lett. 78 (1997) 4865-4968.

[4] J. Nogués, Correlation between antiferromagnetic interface coupling and positive exchange bias, Phys. Rev. B 61 (2000) 1315-1317.

[5] J. Nogués, I. K. Schuller, Exchange bias, J. Magn. Magn. Mater. 192 (1999) 203-232. 
[6] K. Yoshii, Positive exchange bias from magnetization reversal in $\mathrm{La}_{1-\mathrm{x}} \mathrm{Pr}_{\mathrm{x}} \mathrm{CrO}_{3}(\mathrm{x} \sim$ 0.7-0.85), Appl. Phys. Lett. 99 (2011) 142501.

[7] M. Taheri, R. K. Kremer, S. Trudel, F. S. Razavi, Exchange bias effect and glassy-like behavior of $\mathrm{EuCrO}_{3}$ and $\mathrm{CeCrO}_{3}$ nano-powders, Appl. Phys. Lett. 118 (2015) 124306.

[8] P. D. Kulkarni, A. Thamizhavel, V. C. Rakhecha, A. K. Nigam, P. L. Paulose, S. Ramakrishnan, A. K. Grover, Magnetic compensation phenomenon and the sign reversal in the exchange bias field in a single crystal of $\mathrm{Nd}_{0.75} \mathrm{Ho}_{0.25} \mathrm{Al}_{2}$, Europhys. Lett. $86(2009) 47003$.

[9] T. Bora, and S. Ravi, Sign reversal of magnetization and exchange bias field in $\mathrm{LaCr}_{0.85} \mathrm{Mn}_{0.15} \mathrm{O}_{3}$, J. Appl. Phys. 114 (2013) 183902.

[10]R. Shukla, A. K. Bera, S. M. Yusuf, S. K. Deshpande, A. K. Tyagi, W. Hermes, M. Eul, R. Pottgen, Multifunctional Nanocrystalline $\mathrm{CeCrO}_{3}$ : Antiferromagnetic, Relaxor, and Optical Properties, J. Phys. Chem. C 113 (2009) 12663-12668.

[11]Y.M. Cao, S.X. Cao, W. Ren, Z. J. Feng, S. J. Yuan, B. J. Kang, B. Lu, J. C Zhang, Magnetization switching of rare earth orthochromite $\mathrm{CeCrO}_{3}$, Appl. Phys. Lett. 104 (2014) 232405.

[12]L. Wang, S.W. Wang, X. Zhang, L.L. Zhang, R. Yao, G.H. Rao, Reversals of magnetization and exchange-bias in perovskite chromite $\mathrm{YbCrO}_{3}$, J. Alloys Comp. 662 (2016) 268-271.

[13]T. Tamaki, K. Tsushima, Spin Reorientation in $\mathrm{TmCrO}_{3}$, 86-88B (1977) 923-924.

[14]K. Yoshii, Magnetization reversal in $\mathrm{TmCrO}_{3}$, Mater. Res. Bull. 47 (2012) 3243-3248.

[15]D. J. Webb, A. F. Marshall, Z. Sun, T. H. Geballe, and R. M. White, Coercivity of a 
Macroscopic Ferrimagnet Near a Compensation Point, IEEE Trans. Magn. 24 (1988) 588-592.

[16]I. L. Prejbeanu, M Kerekes, R. C. Sousa, H. Sibuet, O. Redon, B. Dieny, and J. P. Nozieres, Thermally assisted MRAM, J. Phys.: Condens. Matter 19 (2007) 165218.

[17]J. C. S. Kools, Exchange-Biased Spin-Valves for Magnetic Storage, IEEE Trans. Magn. 32 (1996) 3165-3184.

\begin{tabular}{cccccc}
\hline & $\mathrm{x}$ & $\mathrm{y}$ & $\mathrm{z}$ & $B_{\text {iso }}\left(\AA^{2}\right)$ & Occ \\
$\operatorname{Tm}(4 \mathrm{c})$ & $0.98199(7)$ & $0.06801(6)$ & 0.25 & $0.792(7)$ & 0.5 \\
$\mathrm{Cr}(4 \mathrm{~b})$ & 0 & 0.5 & 0 & $0.827(17)$ & 0.5 \\
$\mathrm{O} 1(4 \mathrm{c})$ & $0.1120(6)$ & $0.4592(7)$ & 0.25 & $0.52(4)$ & 0.5 \\
$\mathrm{O} 2(8 \mathrm{~d})$ & $0.6924(5)$ & $0.3023(5)$ & $0.0557(3)$ & $0.49(4)$ & 1 \\
\hline lattice parameters $(\AA):$ & $\mathrm{a}=5.19672(6)$ & $\mathrm{b}=5.49182(6) \mathrm{c}=7.48290(4)$ \\
Bragg R-factor= 4.41, Rf- factor=4 .36 \\
Rp= 15.8, Rwp= 17.9 \\
$\chi^{2}=2.64$
\end{tabular}

Table 1. Structural parameters obtained from the Rietveld refinement of XRD pattern of $\mathrm{TmCrO}_{3}$ with the space group Pbnm. 


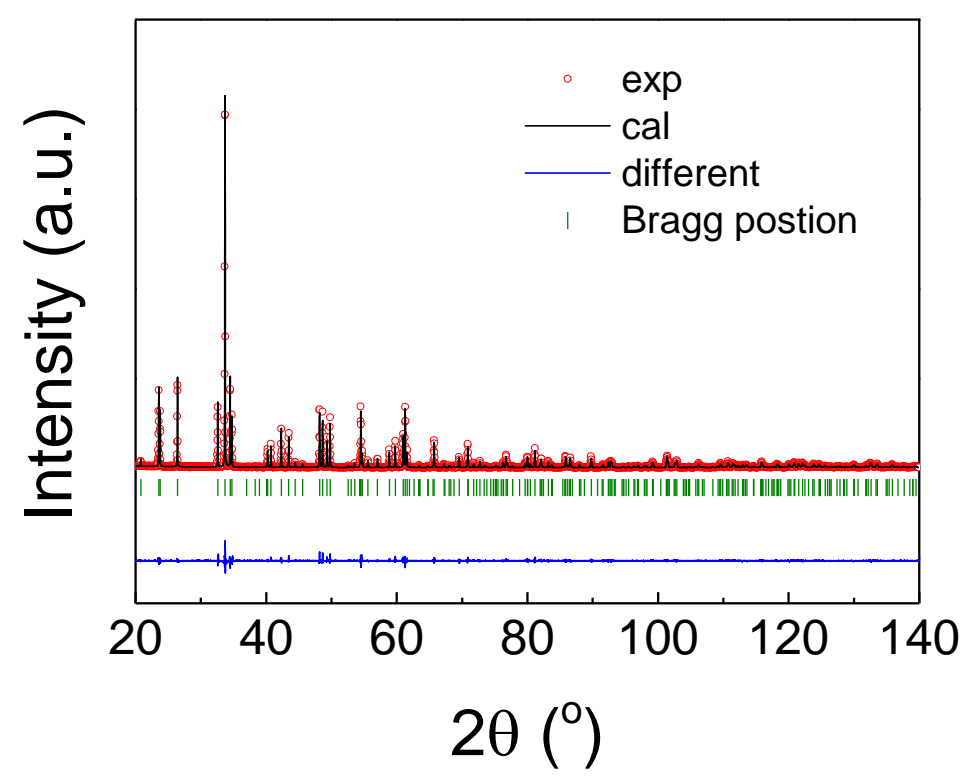

Figure 1. Rietveld refinement results of the XRD pattern of $\mathrm{TmCrO}_{3}$

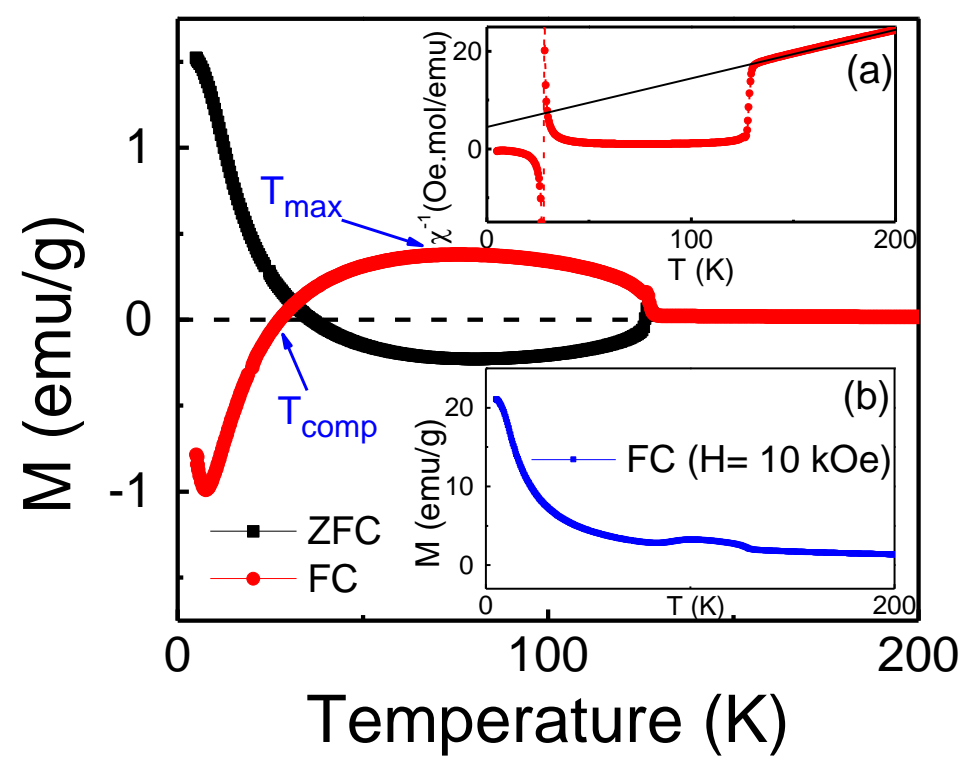

Figure 2. Magnetization-temperature $(M \sim T)$ curve of $\mathrm{TmCrO}_{3}$ measured in $\mathrm{ZFC}$ and $\mathrm{FC}$ processes under the applied field $(\mathrm{H})$ of $100 \mathrm{Oe}$, the inset (a) shows the reciprocal of dc magnetic susceptibility vs temperature, the inset (b) shows the $M \sim T$ curve measured under an applied field of $10 \mathrm{kOe}$. 
(a)

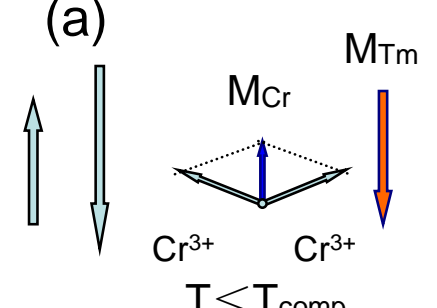

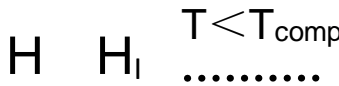

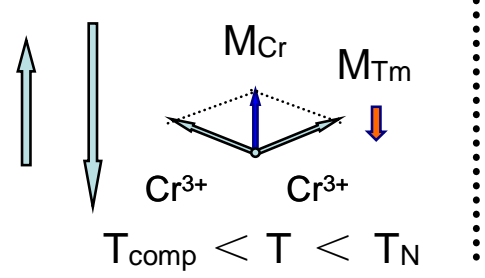

(b)<smiles>C=CC=CC(C)(C)C</smiles>

$\mathrm{H} \quad \mathrm{H}_{1}$ Low cooling field

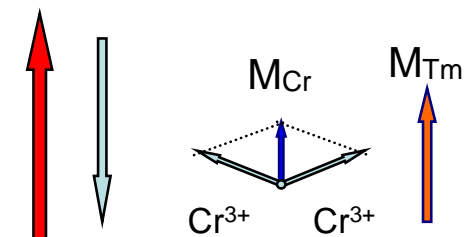

High cooling field

Figure 3. The scheme diagrams of the $\mathrm{Cr}^{3+}$ moments $\left(\mathrm{M}_{\mathrm{Cr}}\right)$ and the $\mathrm{Tm}^{3+}$ moments $\left(\mathrm{M}_{\mathrm{Tm}}\right)$ at different temperature regions (a) and at low/high cooling field (b).

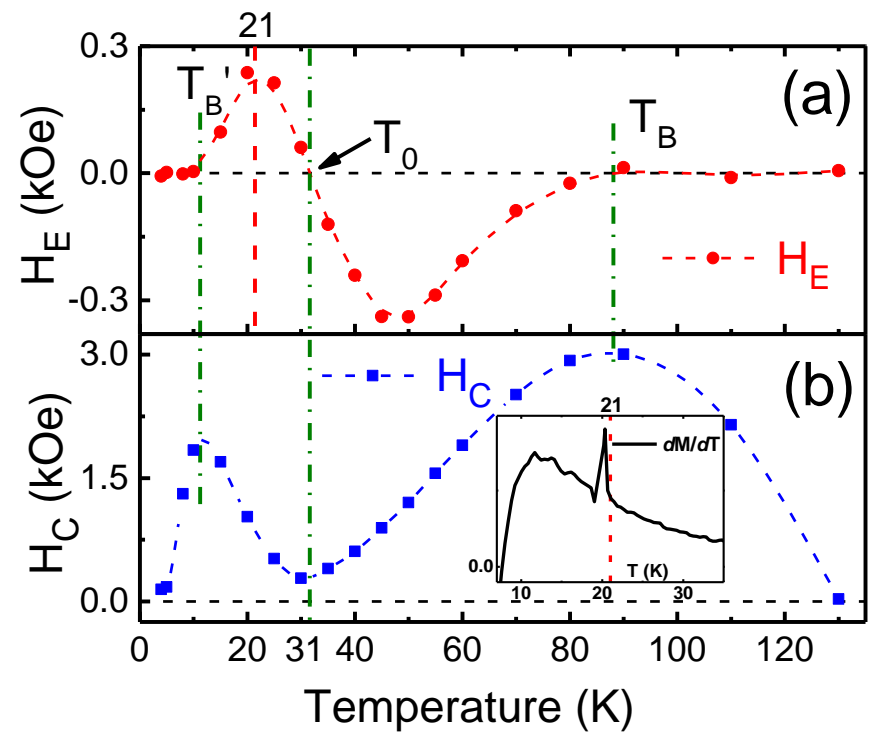

Figure 4. The temperature dependence of $\mathrm{H}_{\mathrm{E}}$ (a) and $\mathrm{H}_{\mathrm{C}}$ (b) measured with a cooling field of $10 \mathrm{kOe}$; the inset shows the first derivative of magnetic susceptibility vs temperature. 


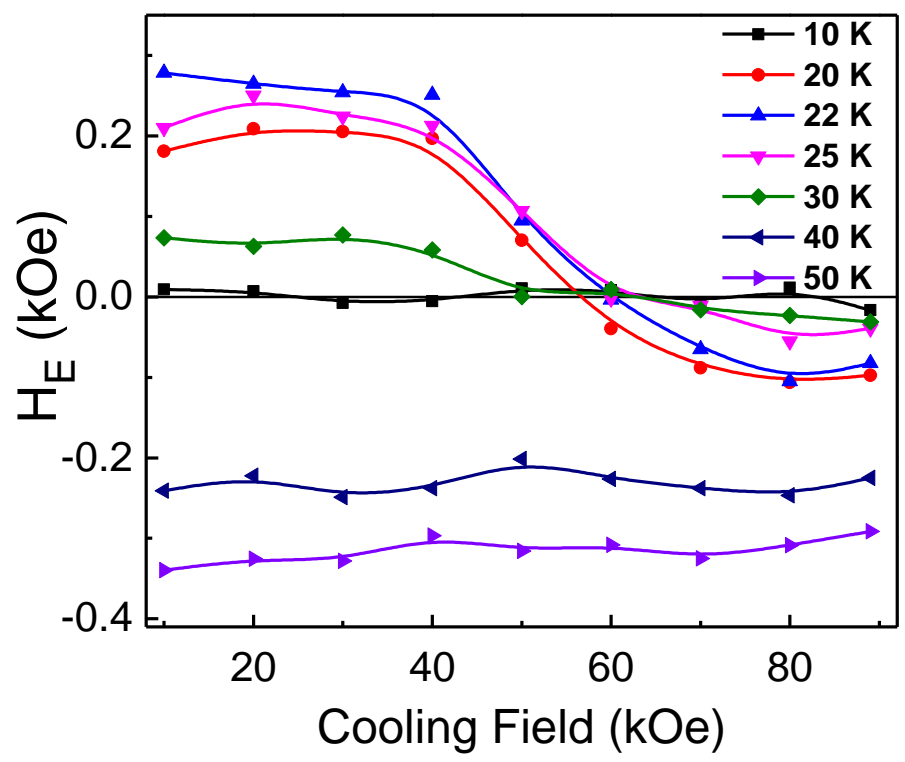

Figure 5. The cooling field dependence of $\mathrm{H}_{\mathrm{E}}$ at different temperature. 